\title{
Existence Results for Solutions of Integral Boundary Value Problems on Time Scales
}

\author{
Erbil Cetin and F. Serap Topal \\ Department of Mathematics, Ege University, Bornova, 35100 Izmir, Turkey \\ Correspondence should be addressed to Erbil Cetin; erbil.cetin@ege.edu.tr
}

Received 27 November 2012; Accepted 12 March 2013

Academic Editor: Xinan Hao

Copyright (C) 2013 E. Cetin and F. S. Topal. This is an open access article distributed under the Creative Commons Attribution License, which permits unrestricted use, distribution, and reproduction in any medium, provided the original work is properly cited.

\begin{abstract}
This paper deals with the existence of solutions for integral boundary value problems (IBVPs) on time scales. We provide sufficient conditions for the existence of solutions by using Schauder fixed point theorem in a cone. Existence result for this problem is also given by the method of upper and lower solutions.
\end{abstract}

\section{Introduction}

The study of dynamic equations on time scales goes back to its founder Hilger [1]. The main motive of the subject of dynamic equations on time scales is that they build bridges between continuous and discrete cases. We begin by presenting some basic definitions on time scale calculus.

A time scale $\mathbb{T}$ is a nonempty closed subset of $\mathbb{R}$. It follows that the jump operators $\sigma, \rho: \mathbb{T} \rightarrow \mathbb{T}$,

$$
\begin{gathered}
\sigma(t)=\inf \{s \in \mathbb{T}: s>t\}, \\
\rho(t)=\sup \{s \in \mathbb{T}: s<t\}
\end{gathered}
$$

are well defined. The point $t \in \mathbb{T}$ is left-dense, left-scattered, right-dense, and right-scattered if $\rho(t)=t, \rho(t)<t, \sigma(t)=t$, and $\sigma(t)>t$, respectively. If $\mathbb{T}$ has a right-scattered minimum $m$, define $\mathbb{T}_{\kappa}=\mathbb{T}-m$; otherwise, set $\mathbb{T}_{\kappa}=\mathbb{T}$. If $\mathbb{T}$ has a leftscattered maximum $M$, define $\mathbb{T}^{\kappa}=\mathbb{T}-M$; otherwise, set $\mathbb{T}^{\mathcal{K}}=\mathbb{T}$. A function $f: \mathbb{T} \rightarrow \mathbb{R}$ is ld-continuous provided it is continuous at left dense points in $\mathbb{T}$, and its right-sided limit exists at right dense points in $T$. For $f: \mathbb{T} \rightarrow \mathbb{R}$ and $t \in \mathbb{T}^{\kappa}$, the delta derivative of $f$ at $t$, denoted by $f^{\Delta}(t)$, is the number with the property that given any $\epsilon>0$, there is a neighborhood $U \subset \mathbb{T}$ of $t$ such that

$$
\left|f(\sigma(t))-f(s)-f^{\Delta}(t)[\sigma(t)-s]\right| \leq \epsilon|\sigma(t)-s|
$$

for all $s \in U$. For $f: \mathbb{T} \rightarrow \mathbb{R}$ and $t \in \mathbb{T}_{\mathcal{K}}$, the nabla derivative of $f$ at $t$, denoted by $f^{\nabla}(t)$, is the number with the property that given any $\epsilon>0$, there is a neighborhood $U \subset \mathbb{V}$ of $t$ such that

$$
\left|f(\rho(t))-f(s)-f^{\nabla}(t)[\rho(t)-s]\right| \leq \epsilon|\rho(t)-s|
$$

for all $s \in U$.

A function $F: \mathbb{T} \rightarrow \mathbb{R}$ is called a nabla antiderivative of $f: \mathbb{T} \rightarrow \mathbb{R}$ provided that $F^{\nabla}(t)=f(t)$ holds for all $t \in \mathbb{T}_{\kappa}$. We then define the nabla integral of $f$ by

$$
\int_{a}^{t} f(s) \nabla s=F(t)-F(a) \quad \forall a, t \in \mathbb{T} .
$$

For the details of basic notions connected to time scales, we refer the readers to the books $[2,3]$ and the papers [4, $5]$, which are useful references for calculus on time scales. Hereafter, we use the notation $[a, b]_{\mathbb{T}}$ to indicate the time scale interval $[a, b] \cap \mathbb{T}$. The intervals $[a, b)_{\mathbb{T}},(a, b]_{\mathbb{T}}$, and $(a, b)_{\mathbb{T}}$ are similarly defined.

Let $\mathbb{T}$ be a time scale such that $a \in\left([a, b]_{\mathbb{T}}\right)_{\kappa}$ and $b \in$ $\left([a, b]_{\pi}\right)^{\kappa}$. We are concerned with existence of solutions of the following integral boundary value problem (IBVP):

$$
\begin{aligned}
-\left[p(t) x^{\Delta}(t)\right]^{\nabla}+q(t) x(t) & =f(t, x(t)), \quad t \in[a, b]_{\mathbb{T}}, \\
\alpha x(\rho(a))-\beta x^{[\Delta]}(\rho(a)) & =\int_{\rho(a)}^{b} h_{1}(x(s)) \nabla s \\
\gamma x(b)+\delta x^{[\Delta]}(b) & =\int_{\rho(a)}^{b} h_{2}(x(s)) \nabla s
\end{aligned}
$$


where $f:[\rho(a), \sigma(b)] \times \mathbb{R} \rightarrow \mathbb{R}$ and $h_{i}: \mathbb{R} \rightarrow \mathbb{R}$ are continuous, $p(t)>0, q(t)>0, \alpha, \beta, \gamma, \delta \geq 0, \alpha+\beta>0$, and $\gamma+\delta>0$, and $x^{[\Delta]}(t)=p(t) x^{\Delta}(t)$.

We would like to mention some results of Khan [6], Yang [7], Ahmad et al. [8], and Atici and Guseinov [9] which motivate us to consider the problem (5)-(7). In [6], Khan considered the method of quasilinearization for the nonlinear boundary value problem with integral boundary conditions

$$
\begin{gathered}
x^{\prime \prime}(t)=f(t, x), \quad t \in[0,1], \\
x(0)-k_{1} x^{\prime}(0)=\int_{0}^{1} h_{1}(x(s)) d s, \\
x(1)+k_{2} x^{\prime}(1)=\int_{0}^{1} h_{2}(x(s)) d s,
\end{gathered}
$$

where $f:[0,1] \times \mathbb{R} \rightarrow \mathbb{R}$ and $h_{i}: \mathbb{R} \rightarrow \mathbb{R}(i=1,2)$ are continuous functions and $k_{i}$ are nonnegative constants. He obtained some results for the existence of solutions in an ordered interval generated by the lower and upper solutions of the boundary value problem. Our work will extend some known results which Khan obtained in [6] for integral boundary value problems to any time scales.

Boundary value problems with integral boundary conditions constitute a very interesting and important class of problems. Various problems in heat conduction, chemical engineering, underground water flow, thermoelasticity, population dynamics, and plasmaphysics [8, 10-12] can be reduced to the problems with integral boundary conditions. For more details of boundary value problems involving integral boundary conditions, see, for instance, $[6,13-19]$ and references therein. Also this type of problems includes twopoint, three-point, and multipoint boundary value problems as special cases $[4,5,7,20]$ and the references therein.

In this section, we obtain some inequalities needed later for certain Green's function. In Section 2, the main tool used in the proof of existence of solutions for the IBVP (5)-(7), is a fixed point theorem in a cone, result due to Schauder [21]. Besides this, in this section, we prove the existence of solutions which will lie between the lower and upper solutions when the lower solution is under the upper solution.

To obtain a solution for the IBVP (5)-(7), we need a mapping whose kernel $G(t, s)$ is the Green's function of the equation

$$
-\left[p(t) x^{\Delta}(t)\right]^{\nabla}+q(t) x(t)=g(t), \quad t \in[a, b]_{\mathbb{T}}
$$

with the integral boundary conditions (6)-(7).

In [9], Atici and Guseinov have shown that the solution $x(t)$ of the nonhomogeneous equation (9) with the nonhomogeneous boundary condition

$$
\begin{array}{r}
\alpha x(\rho(a))-\beta x^{[\Delta]}(\rho(a))=d_{1}, \\
\gamma x(b)+\delta x^{[\Delta]}(b)=d_{2}
\end{array}
$$

is given by

$$
x(t)=w(t)+\frac{1}{D} \int_{\rho(a)}^{b} G(t, s) g(s) \nabla s,
$$

where

$$
\begin{aligned}
w(t) & =\frac{1}{D}\left\{\theta(t) d_{2}-\varphi(t) d_{1}\right\}, \\
D & =-W_{t}[\theta, \varphi] \\
& =\varphi(t) \theta^{[\Delta]}(t)-\varphi^{[\Delta]}(t) \theta(t) .
\end{aligned}
$$

Since the Wronskian of two solutions of the corresponding homogeneous equation

$$
-\left[p(t) x^{\Delta}(t)\right]^{\nabla}+q(t) x(t)=0
$$

under the initial conditions

$$
\begin{array}{cl}
\theta(\rho(a)) & =\beta, \quad \theta^{[\Delta]}(\rho(a))=\alpha, \\
\varphi(b) & =\delta, \quad \varphi^{[\Delta]}(b)=-\gamma
\end{array}
$$

is independent of $t \in[\rho(a), \sigma(b)]_{\mathbb{T}}$, taking $t=\rho(a)$ and $t=b$ in (13), we find $D=\alpha \varphi(\rho(a))-\beta \varphi^{[\Delta]}(\rho(a))=\delta \theta^{[\Delta]}(b)+$ $\gamma \theta(b) \neq 0$, where $\theta(t)$ and $\varphi(t)$ are the linearly independent solutions of (14) subject to conditions (15).

In our problem, we can easily see that the solution of the $\operatorname{IBVP}(5)-(7)$ is

$$
x(t)=P(t)+\frac{1}{D} \int_{\rho(a)}^{b} G(t, s) g(s) \nabla s,
$$

where

$$
\begin{aligned}
P(t)=\frac{1}{D}\{ & \theta(t) \int_{\rho(a)}^{b} h_{2}(x(s)) \nabla s \\
& \left.\quad-\varphi(t) \int_{\rho(a)}^{b} h_{1}(x(s)) \nabla s\right\} .
\end{aligned}
$$

The Green's function in this formula is

$$
G(t, s)= \begin{cases}\theta(s) \varphi(t), & s \leq t \\ \theta(t) \varphi(s), & s \geq t\end{cases}
$$

Lemma 1. Let $\theta(t)$ and $\varphi(t)$ be the solutions of (14) under conditions (15). Then, $\theta(t)$ is strictly increasing and positive on $[\rho(a), \sigma(b)]_{\mathbb{T}}$, and $\varphi(t)$ is strictly decreasing and positive on $[\rho(a), b]_{\mathbb{T}}$.

Proof. In [9], it is shown by Lemma 5.1 that the solutions $\theta$ and $\varphi$ of the BVP (14)-(15) possess the following properties:

$$
\begin{gathered}
\theta(t)>0, \quad \forall t \in(\rho(a), \sigma(b)]_{\mathbb{T}}, \\
\varphi(t)>0, \quad \forall t \in[\rho(a), b)_{\mathbb{T}} .
\end{gathered}
$$

Suppose that there exists at least one $r \in(\rho(a), \sigma(b)]_{\mathbb{T}}$ such $\theta^{\Delta}(r) \leq 0$. From (19) and (14), we obtain

$$
\left[p(t) \theta^{\Delta}(t)\right]^{\nabla}=q(t) \theta(t)>0,
$$


and integrating over $[\rho(a), r]_{\mathbb{T}}$, we get

$$
p(r) \theta^{\Delta}(r)=p(\rho(a)) \theta^{\Delta}(\rho(a))+\int_{\rho(a)}^{r} q(t) \theta(t) \nabla t .
$$

Since $p(\rho(a))>0, \theta^{\Delta}(\rho(a)) \geq 0, q(t)>0$, and $\theta(t)>0$, we obtain $p(r) \theta^{\Delta}(r)>0$. Thus, we determine $\theta^{\Delta}(r)>0$. This contradiction shows that the solution $\theta(t)$ is strictly increasing and positive on $[\rho(a), \sigma(b)]_{\mathbb{T}}$ as desired. Similar arguments can be applied for the proof of $\varphi^{\Delta}(t)<0$ and $\varphi(t)>0$ on $[\rho(a), b]_{\mathbb{T}}$.

Lemma 2. The Green's function $G(t, s)$ defined by (18) satisfies the inequality $G(t, s)=G(s, t)>0$, for all $(t, s) \in[\rho(a), b]_{\mathbb{T}} \times$ $[\rho(a), b]_{\mathbb{T}}$ and $G(t, s) \leq \theta(\sigma(b)) \varphi(\rho(a))$.

Lemma 3. Let $G(t, s)$ be defined by (14). Then, there results are

$$
G(t, s) \geq h(t) G\left(t_{0}, s\right), \quad t, t_{0}, s \in(\rho(a), b)_{\mathbb{T}},
$$

where

$$
\begin{aligned}
h(t) & =\frac{1}{M} \min \{\theta(t), \varphi(t)\}, \\
M & =\max \{\theta(\sigma(b)), \varphi(\rho(a))\} .
\end{aligned}
$$

Proof. For this purpose, we have four cases which are $t, t_{0} \leq s$, $t, t_{0} \geq s, t \leq s \leq t_{0}$, and $t_{0} \leq s \leq t$. We consider only two cases; the others can be shown similarly.

Case 1 . Let $t_{0} \leq s \leq t$. By using Lemma 1 , we get

$$
\begin{aligned}
\frac{G(t, s)}{G\left(t_{0}, s\right)} & \geq \frac{\varphi(t) \theta(s)}{\theta(s) \varphi\left(t_{0}\right)} \geq \frac{\varphi(t)}{\varphi\left(t_{0}\right)} \\
& \geq \frac{\varphi(t)}{\varphi(\rho(a))} \geq \frac{\min \{\theta(t), \varphi(t)\}}{M}=h(t) .
\end{aligned}
$$

Case 2. Let $t_{0} \leq t \leq s$ and $t \leq t_{0} \leq s$. By using Lemma 1, we get

$$
\begin{aligned}
\frac{G(t, s)}{G\left(t_{0}, s\right)} & =\frac{\theta(t) \varphi(s)}{\theta\left(t_{0}\right) \theta(s)} \geq \frac{\theta(t) \varphi\left(t_{0}\right)}{\theta(\sigma(b)) \varphi\left(t_{0}\right)} \\
& =\frac{\theta(t)}{\theta(\sigma(b))} \geq \frac{\min \{\theta(t), \varphi(t)\}}{M}=h(t) .
\end{aligned}
$$

From these cases, we hold $G(t, s) \geq h(t) G\left(t_{0}, s\right)$.

Also, we get

$$
\begin{aligned}
&|P(t)| \leq \mid \frac{1}{D}\left\{\theta(t) \int_{\rho(a)}^{b} h_{2}(x(s)) \nabla s\right. \\
&\left.+\varphi(t) \int_{\rho(a)}^{b} h_{1}(x(s)) \nabla s\right\} \mid \\
& \leq \frac{M}{D} \int_{\rho(a)}^{b}\left|h_{1}(x(s))\right|+\left|h_{2}(x(s))\right| \nabla s:=R .
\end{aligned}
$$

\section{Existence of Solutions}

We will consider the Banach space $B=\{x: x \in C[\rho(a)$, $\left.\sigma(b)]_{\mathbb{T}}\right\}$, with the norm $\|x\|=\max _{t \in[\rho(a), \sigma(b)]_{\mathbb{T}}}|x(t)|$.

Theorem 4. Assume that the function $f(t, \xi)$ is continuous with respect to $\xi \in R$. If $N>0$ satisfies

$$
D R+M^{2} Q(b-\rho(a)) \leq N D,
$$

where $Q>0$ satisfies

$$
Q \geq \max _{\|x\| \leq N}|f(t, x)|, \quad \text { for } t \in[\rho(a), b]_{\mathbb{T}},
$$

then the IBVP (5)-(7) has a solution $x(t)$.

Proof. Let $K:=\{x \in B:\|x\| \leq N\}$. Note that $K$ is closed, bounded, and convex subset of $B$ to which the Schauder fixed point theorem is applicable. Define $A: K \rightarrow B$ by

$$
A x(t):=P(t)+\frac{1}{D} \int_{\rho(a)}^{b} G(t, s) f(s, x(s)) \nabla s,
$$

for $t \in[\rho(a), b]_{\mathbb{T}}$. Obviously, the solutions of problem (5)-(7) are the fixed points of operator $A$. In view of the continuity of the function $f(t, x(t))$, it follows that $A: K \rightarrow B$ is continuous.

Now, we show that $A: K \rightarrow K$. Let $x \in K$. Consider

$$
\begin{aligned}
|A x(t)| & =\left|P(t)+\frac{1}{D} \int_{\rho(a)}^{b} G(t, s) f(s, x(s)) \nabla s\right| \\
& \leq|P(t)|+\frac{1}{D} \int_{\rho(a)}^{b}|G(t, s)||f(s, x(s))| \nabla s \\
& \leq R+\frac{M^{2}}{D} \int_{\rho(a)}^{b}|f(s, x(s))| \nabla s \\
& \leq R+\frac{M^{2} Q(b-\rho(a))}{D} \leq N
\end{aligned}
$$

for every $t \in[\rho(a), b]_{\mathbb{T}}$. This implies that $\|A x\| \leq N$.

Thus, all functions which belong to $A(K)$ are equibounded and $A(K) \subset K$. The uniform continuity of the $G(t, s)$ and $f(t, x)$ implies that all functions in $A(K)$ are equicontinuous. So, by Arzela-Ascoli theorem, the operator $A$ : $K \rightarrow K$ is compact. Hence, $A$ has a fixed point in $K$ by Schauder fixed point theorem.

Corollary 5. If $f$ is continuous and bounded on $[\rho(a), b]_{\mathbb{T}} \times \mathbb{R}$, then the IBVP (5)-(7) has a solution.

Let us define the set $S$ by

$S:=\left\{x: x^{\Delta}\right.$ is continuous on $[\rho(a), b]_{\mathbb{T}}$ and $p x^{\Delta}$

is nabla differentiable on $[a, b]_{\mathbb{T}}$ and $\left(p x^{\Delta}\right)^{\nabla}$

is ld-continuous on $\left.[a, b]_{\mathbb{T}}\right\}$.

For any $u, v \in S$, we define the sector $[u, v]$ by

$$
[u, v]:=\{w \in E: u \leq w \leq v\} .
$$


Definition 6. A real valued function $u(t) \in S$ on $[\rho(a), b]_{\mathbb{T}}$ is a lower solution for IBVP (5)-(7) if

$$
\begin{gathered}
-\left[p(t) u^{\Delta}(t)\right]^{\nabla}+q(t) u(t) \leq f(t, u(t)), \quad t \in[a, b]_{\mathbb{T}} \\
\alpha u(\rho(a))-\beta u^{[\Delta]}(\rho(a)) \leq \int_{\rho(a)}^{b} h_{1}(u(s)) \nabla s \\
\gamma u(b)+\delta u^{[\Delta]}(b) \leq \int_{\rho(a)}^{b} h_{2}(u(s)) \nabla s .
\end{gathered}
$$

Similarly, real valued function $v(t) \in S$ on $[\rho(a), b]_{\mathbb{T}}$ is an upper solution for IBVP (5)-(7) if the inequalities in (34) are satisfied in the reverse direction for $v(t)$.

Theorem 7. Assume that $u$ and $v$ are, respectively, lower and upper solutions of (5)-(7). If $f:[\rho(a), \sigma(b)]_{\mathbb{T}} \times \mathbb{R} \rightarrow \mathbb{R}$ is continuous, $h_{i}: \mathbb{R} \rightarrow \mathbb{R}(i=1,2)$ are continuously differentiable, $f(t, x)$ is decreasing in $x$ for $t \in[\rho(a), b]_{\mathbb{T}}$, and $0 \leq h_{1}^{\prime}(x)<\alpha /(b-\rho(a))$, then

$$
u(t) \leq v(t), \quad t \in[\rho(a), b)_{\mathbb{T}} .
$$

Proof. Define $g:=u-v$. For the sake of contradiction, assume that the result is not true on $[\rho(a), b)_{\mathbb{T}}$. Then, the function $g(t)$ has a positive maximum at $t_{0} \in(\rho(a), b)_{\mathbb{T}}$ and $(u-v)(t)<$ $(u-v)\left(t_{0}\right)$ for $t \in\left(t_{0}, b\right)_{\mathbb{T}}$. So, we have $g\left(t_{0}\right)>0, g^{\Delta}\left(t_{0}\right) \leq 0$, and $\left(p g^{\Delta}\right)^{\nabla}\left(t_{0}\right) \leq 0$; hence,

$$
\begin{aligned}
-\left[p\left(t_{0}\right) g^{\Delta}\left(t_{0}\right)\right]^{\nabla} & \leq-\left[p\left(t_{0}\right) g^{\Delta}\left(t_{0}\right)\right]^{\nabla}+q\left(t_{0}\right) g\left(t_{0}\right) \\
& =-\left[p\left(t_{0}\right) u^{\Delta}\left(t_{0}\right)\right]^{\nabla}+q\left(t_{0}\right) u\left(t_{0}\right) \\
& -\left\{-\left[p\left(t_{0}\right) v^{\Delta}\left(t_{0}\right)\right]^{\nabla}+q\left(t_{0}\right) v\left(t_{0}\right)\right\} \\
& \leq f\left(t_{0}, u\left(t_{0}\right)\right)-f\left(t_{0}, v\left(t_{0}\right)\right)<0,
\end{aligned}
$$

a contradiction. If $t_{0}=\rho(a)$, then $g(\rho(a))>0$ and $g^{\Delta}(\rho(a)) \leq$ 0 . Using the boundary conditions, we have

$$
\begin{aligned}
\alpha g(\rho(a)) \leq & \beta p(\rho(a)) g^{\Delta}(\rho(a)) \\
& +\int_{\rho(a)}^{b}\left(h_{1}(u(s))-h_{1}(v(s))\right) \nabla s \\
& \leq \int_{\rho(a)}^{b}\left(h_{1}(u(s))-h_{1}(v(s))\right) \nabla s .
\end{aligned}
$$

On the other hand, using the mean value theorem and the assumption on $h_{1}(x)$, we obtain

$$
\begin{aligned}
\alpha g(\rho(a)) & \leq \int_{\rho(a)}^{b} h_{1}^{\prime}(c) g(s) \nabla s \\
& <\frac{\alpha}{b-\rho(a)} \int_{\rho(a)}^{b} g(s) \nabla s \\
& <\frac{\alpha}{b-\rho(a)} \max _{t \in[\rho(a), b)_{\mathbb{T}}} g(t)(b-\rho(a)) \\
& <\alpha g(\rho(a)),
\end{aligned}
$$

where $c \in(u(s), v(s))$, a contradiction. Hence,

$$
u(t) \leq v(t), \quad t \in[\rho(a), b)_{\mathbb{T}} .
$$

Now, we state and prove the existence and uniqueness of solutions in an ordered interval generated by the lower and upper solutions of the boundary value problem.

Theorem 8. Assume that $u$ and $v$ are, respectively, lower and upper solutions of (5)-(7) such that $u(t) \leq v(t), t \in$ $[\rho(a), \sigma(b)]_{\mathbb{T}}$. If $f:[\rho(a), \sigma(b)]_{\mathbb{T}} \times \mathbb{R} \rightarrow \mathbb{R}$ and $h_{i}: \mathbb{R} \rightarrow \mathbb{R}$ $(i=1,2)$ are continuously differentiable and $h_{1}^{\prime}(x) \geq 0$, then there exists a solution $x(t)$ of (5)-(7) such that

$$
u(t) \leq x(t) \leq v(t), \quad t \in[\rho(a), b)_{\mathbb{T}} .
$$

Proof. Define the following modifications of $f(t, x)$ and $h_{i}(x), i=1,2$ :

$$
F(t, x)= \begin{cases}f(t, v(t))+\frac{x-v(t)}{1+|x-v|}, & x \geq v(t) \\ f(t, x(t)), & u(t) \leq x \leq v(t), \\ f(t, u(t))-\frac{x-u(t)}{1+|x-u|}, & x \leq u(t)\end{cases}
$$

for $t \in[\rho(a), \sigma(b)]_{\mathbb{T}}$, and

$$
H_{i}(x)= \begin{cases}h_{i}(v(t)), & x \geq v(t), \\ h_{i}(x(t)), & u(t) \leq x \leq v(t), \\ h_{i}(u(t)), & x \leq u(t) .\end{cases}
$$

Consider the modified problem

$$
\begin{aligned}
-\left[p(t) x^{\Delta}(t)\right]^{\nabla}+q(t) x(t) & =F(t, x(t)), \quad t \in[a, b]_{\mathbb{T}}, \\
\alpha x(\rho(a))-\beta x^{[\Delta]}(\rho(a)) & =\int_{\rho(a)}^{b} H_{1}(x(s)) \nabla s, \\
\gamma x(b)+\delta x^{[\Delta]}(b) & =\int_{\rho(a)}^{b} H_{2}(x(s)) \nabla s .
\end{aligned}
$$


As $F:[\rho(a), \sigma(b)]_{\mathbb{T}} \times \mathbb{R} \rightarrow \mathbb{R}$ and $H_{i}: \mathbb{R} \rightarrow \mathbb{R}(i=1,2)$ are continuous and bounded, it follows that the boundary value problem (43) has a solution. Further, note that

$$
\begin{aligned}
-\left[p(t) u^{\Delta}(t)\right]^{\nabla}+q(t) u(t) & \leq f(t, u(t)) \\
& =F(t, u(t)), \quad t \in[a, b]_{T}, \\
\alpha u(\rho(a))-\beta u^{[\Delta]}(\rho(a)) & \leq \int_{\rho(a)}^{b} h_{1}(u(s)) \nabla s \\
& =\int_{\rho(a)}^{b} H_{1}(u(s)) \nabla s \\
\gamma u(b)+\delta u^{[\Delta]}(b) \leq & \int_{\rho(a)}^{b} h_{2}(u(s)) \nabla s \\
& =\int_{\rho(a)}^{b} H_{2}(u(s)) \nabla s
\end{aligned}
$$

which imply that $u(t)$ is a lower solution of (43). Similarly, $v(t)$ is an upper solution of (43). We need to show that any solution $x(t)$ of (43) is such that $u(t) \leq x(t) \leq v(t), t \in$ $[\rho(a), b)_{\mathbb{T}}$. Assume that $u(t) \leq x(t)$ is not true on $[\rho(a), b)_{\mathbb{T}}$. Then, the function $k(t)=u(t)-x(t)$ has a positive maximum at $t_{0} \in(\rho(a), b)_{\mathbb{T}}$ and $(u-x)(t)<(u-x)\left(t_{0}\right)$ for $t \in\left(t_{0}, b\right)_{\mathbb{T}}$. So, we have $k\left(t_{0}\right)>0, k^{\Delta}\left(t_{0}\right) \leq 0$, and $\left(p k^{\Delta}\right)^{\nabla}\left(t_{0}\right) \leq 0$ by Lemma 6.17 in [3]; hence,

$$
\begin{aligned}
0 & \leq-\left[p\left(t_{0}\right) k^{\Delta}\left(t_{0}\right)\right]^{\nabla} \leq-\left[p\left(t_{0}\right) k^{\Delta}\left(t_{0}\right)\right]^{\nabla}+q\left(t_{0}\right) k\left(t_{0}\right) \\
& =-\left[p\left(t_{0}\right)(u-x)^{\Delta}\left(t_{0}\right)\right]^{\nabla}+q\left(t_{0}\right)(u-x)\left(t_{0}\right) \\
& =-\left[p\left(t_{0}\right) u^{\Delta}\left(t_{0}\right)\right]^{\nabla}+q\left(t_{0}\right) u\left(t_{0}\right) \\
& -\left\{-\left[p\left(t_{0}\right) x^{\Delta}\left(t_{0}\right)\right]^{\nabla}+q\left(t_{0}\right) x\left(t_{0}\right)\right\} \\
& \leq f\left(t_{0}, u\left(t_{0}\right)\right)-\left\{f\left(t_{0}, u\left(t_{0}\right)\right)\right. \\
& \left.-\frac{x\left(t_{0}\right)-u\left(t_{0}\right)}{1+\left|x\left(t_{0}\right)-u\left(t_{0}\right)\right|}\right\}<0,
\end{aligned}
$$

a contradiction. If $t_{0}=\rho(a)$, then $k(\rho(a))>0$ and $k^{\Delta}(\rho(a)) \leq$ 0 , but then the boundary conditions and the nondecreasing property of $h_{i}$ give

$$
\begin{aligned}
\alpha k(\rho(a))= & \alpha(u(\rho(a))-x(\rho(a))) \\
\leq & \beta u^{[\Delta]}(\rho(a))+\int_{\rho(a)}^{b} h_{1}(u(s)) \nabla s-\beta x^{[\Delta]}(\rho(a)) \\
& -\int_{\rho(a)}^{b} H_{1}(x(s)) \nabla s
\end{aligned}
$$

$$
\begin{aligned}
= & \beta p(\rho(a))\left(k^{\Delta}(\rho(a))\right) \\
& +\int_{\rho(a)}^{b}\left(h_{1}(u(s))-H_{1}(x(s))\right) \nabla s \\
\leq & \int_{\rho(a)}^{b}\left(h_{1}(u(s))-H_{1}(x(s))\right) \nabla s .
\end{aligned}
$$

If $x<u(t)$, then $H_{1}(x(s))=h_{1}(u(s))$, and, hence, $\alpha k(\rho(a)) \leq 0$, a contradiction. If $x>v(t)$, then $H_{1}(x(s))=$ $h_{1}(v(s)) \geq h_{1}(u(s))$, which implies $\alpha k(\rho(a)) \leq 0$, a contradiction. Hence, $u(t) \leq x(t) \leq v(t)$ and $H_{1}(x(s))=h_{1}(x(s)) \geq$ $h_{1}(u(s))$ and $\alpha k(\rho(a)) \leq 0$, another contradiction.

We can illustrate our results in the following examples.

Example 9. Let $\mathbb{T}$ be any time scales such that $1 \in\left([0,1]_{\mathbb{T}}\right)^{\kappa}$ and $0 \in\left([0,1]_{\mathbb{T}}\right)_{\kappa}$. We consider the following IBVP:

$$
\begin{gathered}
-x^{\Delta \nabla}(t)=\sin x(t), \quad t \in[0,1]_{\mathbb{T}}, \\
2 x(\rho(0))-x^{\Delta}(\rho(0))=\int_{\rho(0)}^{1} x(s) \nabla s, \\
x(1)-2 x^{\Delta}(1)=\int_{\rho(0)}^{1} x(s) \nabla s .
\end{gathered}
$$

From (15), we have $\theta(t)=2(t-\rho(0))+1$ and $\varphi(t)=-t-1$.

We calculate easily

$$
\begin{aligned}
M & =\max \{\theta(\sigma(1)), \varphi(\rho(0))\} \\
& =\max \{2(\sigma(1)-\rho(0))+1,-\rho(0)-1\} \\
& =2(\sigma(1)-\rho(0))+1, \\
D & =\varphi(t) \theta^{\Delta}(t)-\varphi^{\Delta}(t) \theta(t) \\
& =(-t-1) 2-(-1)(2(t-\rho(0))+1) .
\end{aligned}
$$

For $t=1$ or $t=\rho(0), D=-1-2 \rho(0)$.

We have

$$
\begin{aligned}
|P(t)| & \leq \frac{M}{D} \int_{\rho(0)}^{1}\left[\left|h_{1}(x(s))\right|+\left|h_{2}(x(s))\right|\right] \nabla s \\
& =\frac{2(\sigma(1)-\rho(0))+1}{-1-2 \rho(0)} \int_{\rho(0)}^{1} 2|x(s)| \nabla s \\
& \leq \frac{2(\sigma(1)-\rho(0))+1}{-1-2 \rho(0)} 2 Q(1-\rho(0)):=R,
\end{aligned}
$$

where $Q=\max _{t \in[\rho(0), 1]}|x(t)|$. Since $\max _{\|x\| \leq N}|f(t, x)|=$ $\max _{\|x\| \leq N}|\sin x(t)| \leq \max _{\|x\| \leq N}|x(t)| \leq\|x\| \leq N \leq Q$ for $t \in[\rho(0), 1]$, there exists a positive real number $N$ which satisfies $R+M^{2} Q(1-\rho(0)) / D \leq N$ for the positive $Q$, and then all condition in Theorem 4 are satisfied. Therefore, the IBVP (5)-(7) has a solution $x(t)$. 
Example 10. Let $\mathbb{T}=[0,1 / 4] \cup\{1 / 3\} \cup[1 / 2,2]$. We consider the following IBVP:

$$
\begin{aligned}
-x^{\Delta \nabla}(t)+x(t) & =e^{-x(t)}, \quad t \in[0,1]_{\mathbb{T}}, \\
2 x(\rho(0))-x^{\Delta}(\rho(0)) & =\int_{\rho(0)}^{1} x^{2}(s) \nabla s \\
x(1)+2 x^{\Delta}(1) & =\int_{\rho(0)}^{1} x(s) \nabla s .
\end{aligned}
$$

For $u(t)=-1$, we get

$$
\begin{gathered}
-u^{\Delta \nabla}(t)+u(t)=-1 \leq e^{1}, \quad t \in[0,1]_{\mathbb{T}}, \\
2(-1)-0 \leq \int_{\rho(0)}^{1}(-1)^{2} \nabla s=1 \\
(-1)+0 \leq \int_{\rho(0)}^{1}(-1) \nabla s=-1 .
\end{gathered}
$$

Thus, $u(t)=-1$ is the lower solution.

For $v(t)=1$, we get

$$
\begin{gathered}
-v^{\Delta \nabla}(t)+v(t)=1 \geq e^{-1}, \quad t \in[0,1]_{\mathbb{T}}, \\
2(1)-0 \geq \int_{\rho(0)}^{1}(1)^{2} \nabla s=1 \\
(1)+0 \geq \int_{\rho(0)}^{1}(1) \nabla s=1 .
\end{gathered}
$$

Thus, $v(t)=1$ is the upper solution.

Theorem 8 implies that IBVP has a solution $x(t)$ such that

$$
-1 \leq x(t) \leq 1, \quad t \in[\rho(0), 1)_{\mathbb{T}} .
$$

Example 11. Let $\mathbb{T}$ be any time scales such that $1 \in\left([0,1]_{\mathbb{T}}\right)^{\kappa}$ and $0 \in\left([0,1]_{\mathbb{T}}\right)_{\kappa}$. We consider the following IBVP:

$$
\begin{gathered}
-\left[(t+1) x^{\Delta}(t)\right]^{\nabla}+e^{t} x(t)=\sin x(t)-x(t), \quad t \in[0,1]_{\mathbb{V}}, \\
4(1-\rho(0)) x(\rho(0))-3 t x^{\Delta}(\rho(0))=\int_{\rho(0)}^{1} x^{3}(s) \nabla s, \\
16(1-\rho(0)) x(1)-2 t x^{\Delta}(1)=\int_{\rho(0)}^{1} x^{5}(s) \nabla s .
\end{gathered}
$$

Let $u(t)=0$. We can easily see that $u(t)$ satisfies all conditions of a lower solution. Hence, $u(t)=0$ is the lower solution.

Let $v(t)=\pi / 2$, and

$$
\begin{aligned}
-\left[(t+1) v^{\Delta}(t)\right]^{\nabla}+e^{t} v(t) & =e^{t} \frac{\pi}{2} \geq 1-\frac{\pi}{2} \\
& =\sin v(t)-v(t), \quad t \in[0,1]_{\mathbb{T}} .
\end{aligned}
$$

We get $\left(e^{t}+1\right)(\pi / 2) \geq 1$. From

$$
\begin{aligned}
4(1-\rho(0)) \frac{\pi}{2}-0 & \geq \int_{\rho(0)}^{1}\left(\frac{\pi}{2}\right)^{3} \nabla s \\
& =\left(\frac{\pi}{2}\right)^{3}(1-\rho(0)),
\end{aligned}
$$

we get $16 \geq \pi^{2}$, and from

$$
\begin{aligned}
16(1-\rho(0)) \frac{\pi}{2}-0 & \geq \int_{\rho(0)}^{1}\left(\frac{\pi}{2}\right)^{5}(s) \nabla s \\
& =\left(\frac{\pi}{2}\right)^{5}(1-\rho(0)),
\end{aligned}
$$

we get $256 \geq \pi^{4}$. Thus, $v(t)=\pi / 2$ is the upper solution.

Theorem 8 implies that IBVP has a solution $x(t)$ such that

$$
0 \leq x(t) \leq \frac{\pi}{2}, \quad t \in[\rho(0), 1)_{\mathbb{T}} .
$$

\section{References}

[1] S. Hilger, "Analysis on measure chains-a unified approach to continuous and discrete calculus," Results in Mathematics, vol. 18, no. 1-2, pp. 18-56, 1990.

[2] M. Bohner and A. Peterson, Dynamic Equations on Time Scales: An Introduction with Applications, Birkhäuser, Boston, Mass, USA, 2001.

[3] M. Bohner and A. Peterson, Advances in Dynamic Equations on Time Scales, Birkhäuser, Boston, Mass, USA, 2003.

[4] F. A. Davidson and B. P. Rynne, "The formulation of secondorder boundary value problems on time scales," Advances in Difference Equations, vol. 2006, Article ID 31430, 10 pages, 2006.

[5] L. Erbe and A. Peterson, "Green's functions and comparison theorems for differential equations on measure chains," Dynamics of Continuous, Discrete and Impulsive Systems, vol. 6, no. 1, pp. 121-137, 1999.

[6] R. A. Khan, "The generalized method of quasilinearization and nonlinear boundary value problems with integral boundary conditions," Electronic Journal of Qualitative Theory of Differential Equations, vol. 10, pp. 1-9, 2003.

[7] Z. Yang, "Existence and nonexistence results for positive solutions of an integral boundary value problem," Nonlinear Analysis: Theory, Methods \& Applications, vol. 65, no. 8, pp. 1489-1511, 2006.

[8] B. Ahmad, A. Alsaedi, and B. S. Alghamdi, "Analytic approximation of solutions of the forced Duffing equation with integral boundary conditions," Nonlinear Analysis: Real World Applications, vol. 9, no. 4, pp. 1727-1740, 2008.

[9] F. M. Atici and G. Sh. Guseinov, "On Green's functions and positive solutions for boundary value problems on time scales," Journal of Computational and Applied Mathematics, vol. 141, no. 1-2, pp. 75-99, 2002.

[10] G. W. Batten Jr., "Second-order correct boundary conditions for the numerical solution of the mixed boundary problem for parabolic equations," Mathematics of Computation, vol. 17, pp. 405-413, 1963.

[11] J. R. Cannon, S. P. Esteva, and J. van der Hoek, "A Galerkin procedure for the diffusion equation subject to the specification of mass," SIAM Journal on Numerical Analysis, vol. 24, no. 3, pp. 499-515, 1987. 
[12] L. I. Kamynin, "A boundary value problem in the theory of heat conduction with boundary condition," USSR Computational Mathematics and Mathematical Physics, vol. 4, no. 6, pp. 33-59, 1964.

[13] A. Bouziani and N.-E. Benouar, "Mixed problem with integral conditions for a third order parabolic equation," Kobe Journal of Mathematics, vol. 15, no. 1, pp. 47-58, 1998.

[14] M. Denche and A. L. Marhoune, "Mixed problem with integral boundary condition for a high order mixed type partial differential equation," Journal of Applied Mathematics and Stochastic Analysis, vol. 16, no. 1, pp. 69-79, 2003.

[15] J. M. Gallardo, "Second-order differential operators with integral boundary conditions and generation of analytic semigroups," The Rocky Mountain Journal of Mathematics, vol. 30, no. 4, pp. 1265-1292, 2000.

[16] T. Jankowski, "Differential equations with integral boundary conditions," Journal of Computational and Applied Mathematics, vol. 147, no. 1, pp. 1-8, 2002.

[17] A. V. Kartynnik, "A three-point mixed problem with an integral condition with respect to the space variable for second-order parabolic equations," Differential Equations, vol. 26, no. 9, pp. 1160-1166, 1990.

[18] L. Liu, X. Hao, and Y. Wu, "Positive solutions for singular second order differential equations with integral boundary conditions," Mathematical and Computer Modelling, vol. 57, no. 3-4, pp. 836847, 2013.

[19] X. Zhang and W. Ge, "Symmetric positive solutions of boundary value problems with integral boundary conditions," Applied Mathematics and Computation, vol. 219, no. 8, pp. 3553-3564, 2012.

[20] S. A. Brykalov, "A second-order nonlinear problem with twopoint and integral boundary conditions," Georgian Mathematical Journal, vol. 1, no. 3, pp. 243-249, 1994.

[21] M. A. Krasnosel'skii, Positive Solutions of Operator Equations, P. Noordhoff Ltd., Groningen, The Netherlands, 1964. 


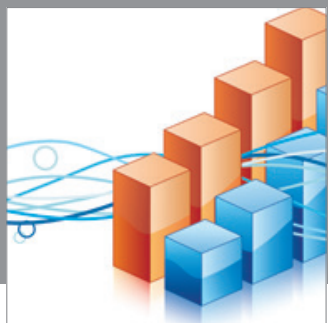

Advances in

Operations Research

mansans

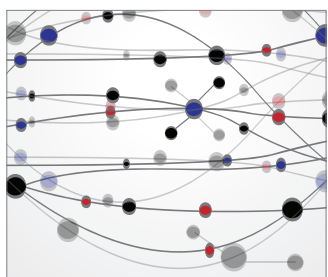

The Scientific World Journal
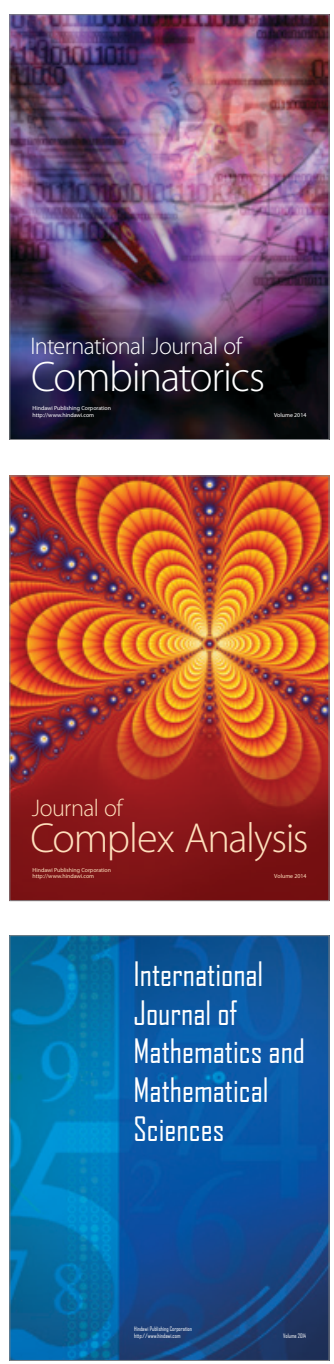
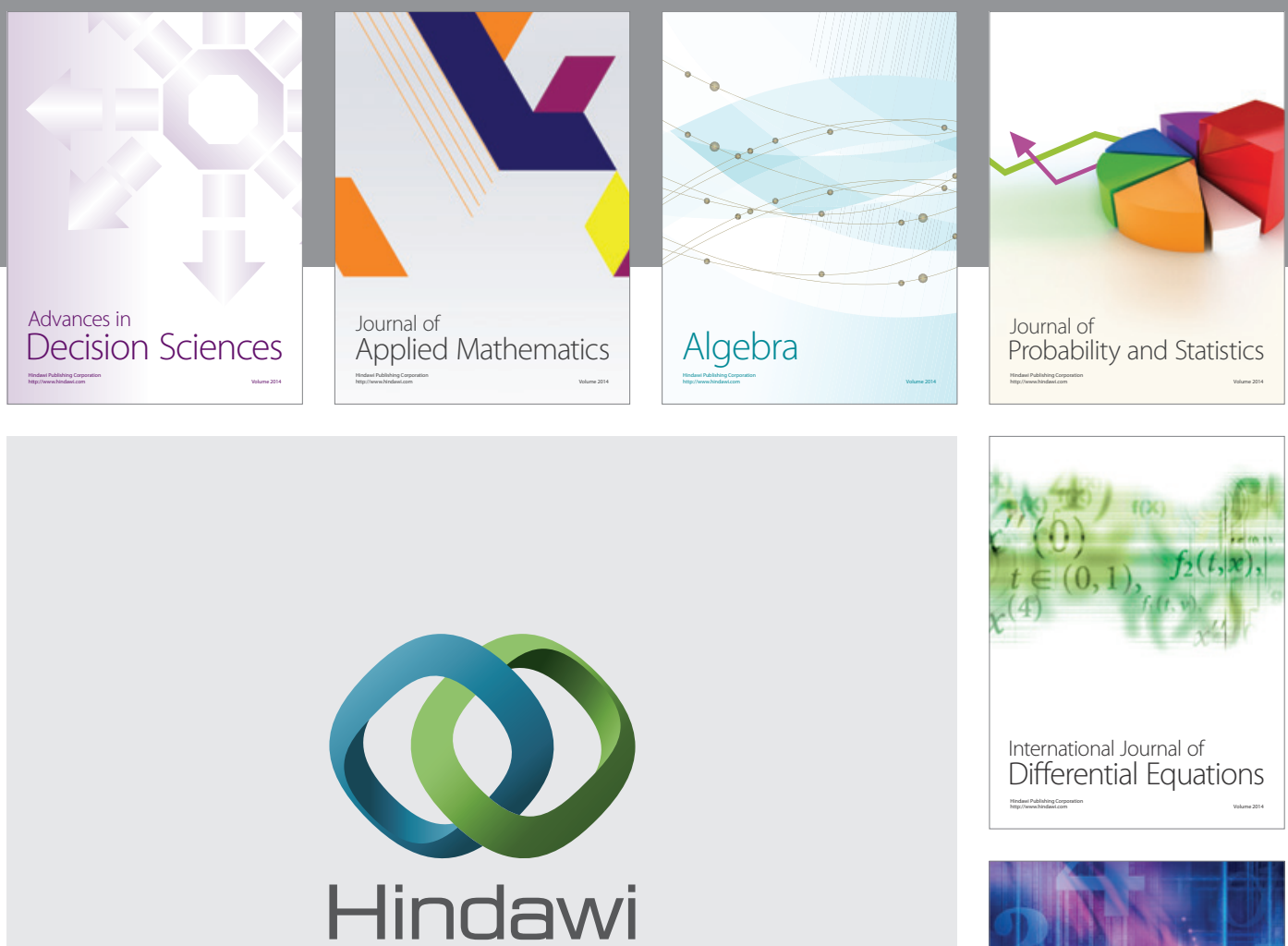

Submit your manuscripts at http://www.hindawi.com
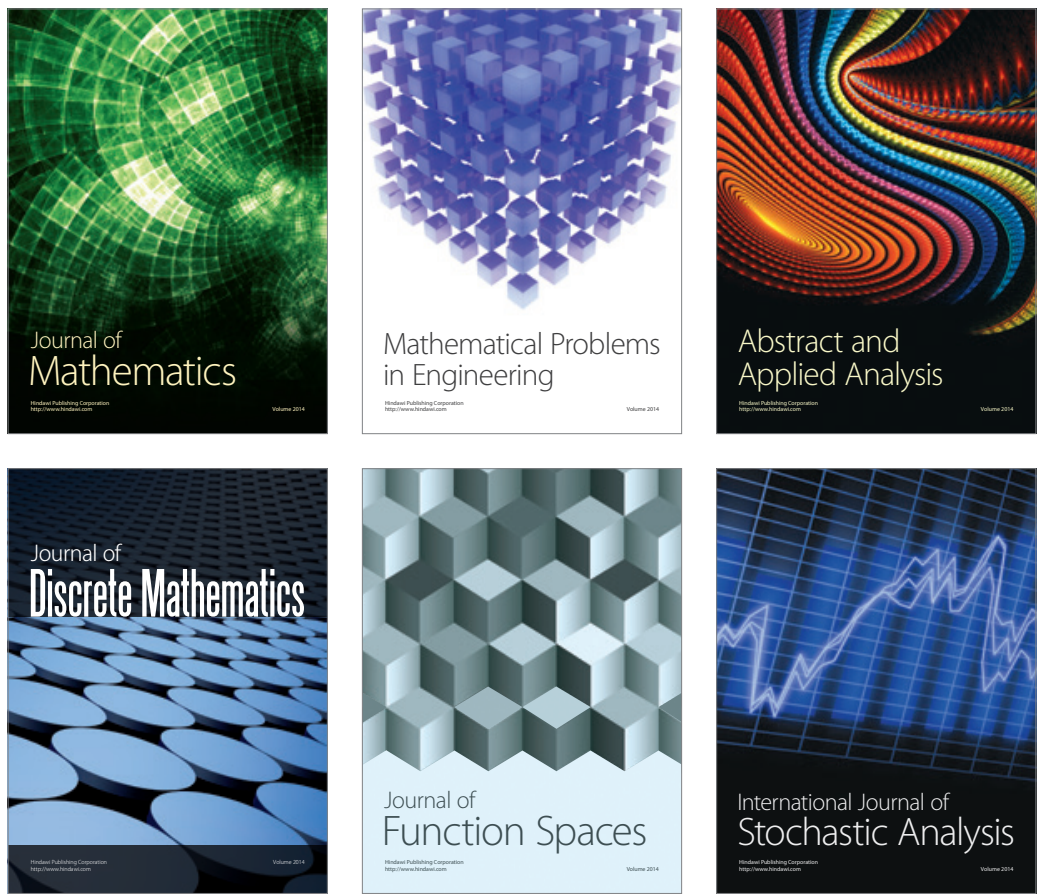

Journal of

Function Spaces

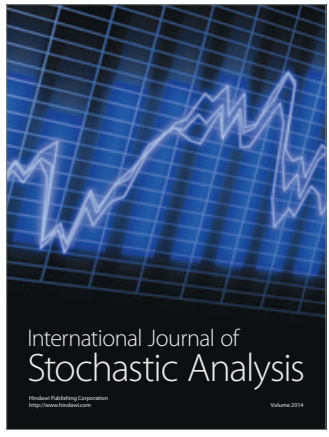

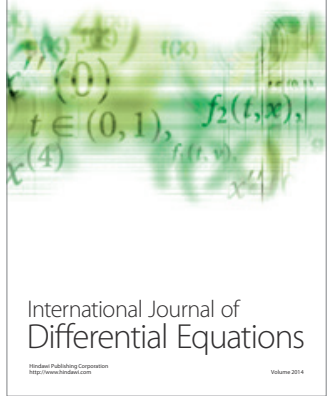
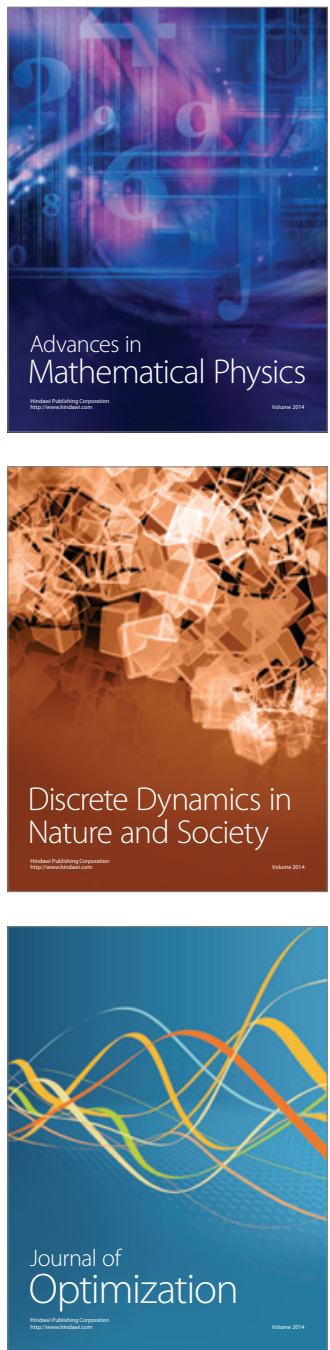